\title{
Design of Telescopic Nadir Imager for Geomorphology (TENGO0) and Observation of Surface Reflectance by Optical Chromatic Imager (OROCHI) for the Martian Moons Exploration (MMX)
}

Shingo Kameda ( $\sim$ kameda@rikkyo.ac.jp)

Rikkyo University https://orcid.org/0000-0001-9084-2858

Masanobu Ozaki

ISAS/JAXA

Keigo Enya

ISAS/JAXA

Ryota Fuse

Nihon University: Nihon Daigaku

Toru Kouyama

AIST

Naoya Sakatani

Rikkyo University: Rikkyo Daigaku

Hidehiko Suzuki

Meiji University: Meiji Daigaku

Naoya Osada

Rikkyo University: Rikkyo Daigaku

Hiroki Kato

Rikkyo University: Rikkyo Daigaku

Hideaki Miyamoto

The University of Tokyo: Tokyo Daigaku

Atsushi Yamazaki

ISAS/JAXA

Tomoki Nakamura

Tohoku University: Tohoku Daigaku

Takaya Okamoto

ISAS/JAXA

Takahiro Ishimaru

ISAS/JAXA

Peng Hong

Chiba Institute of Technology: Chiba Kogyo Daigaku 


\section{Ko Ishibashi}

Chiba Institute of Technology: Chiba Kogyo Daigaku

\section{Takeshi Takashima}

ISAS/JAXA

\section{Ryoya Ishigami}

Wakasawan Energy Kenkyu Center

\section{Cheng Ling Kuo}

National Central University

\section{Shinsuke Abe}

Nihon University: Nihon Daigaku

\section{Yuya Goda}

MEISEI

\section{Hajime Murao}

MEISEI

\section{Saori Fujishima}

Meisei Daigaku - Hino Campus: Meisei Daigaku

\section{Tsubasa Aoyama}

$$
\text { MEISEI }
$$

\section{Keiji Hagiwara}

MEISEI

\section{Satoko Mizumoto}

MEISEI

Noriko Tanaka

MEISEI

\section{Kousuke Murakami}

NIKON

Miho Matsumoto

NIKON

Kenji Tanaka

NIKON

\section{Hironobu Sakuta}

NIKON

\section{Full paper}

Keywords: MMX, TENGOO, OROCHI, imager, Phobos, Deimos, Mars

Posted Date: January 28th, 2021

DOl: https://doi.org/10.21203/rs.3.rs-153778/v1 
License: (c) (i) This work is licensed under a Creative Commons Attribution 4.0 International License. Read Full License

Version of Record: A version of this preprint was published at Earth, Planets and Space on December 1st, 2021. See the published version at https://doi.org/10.1186/s40623-021-01462-9. 


\section{Abstract}

The JAXA's Martian Moons Exploration (MMX) mission is planned to reveal the origin of Phobos and Deimos. Both moons will be observed by remote sensing. Sample return from Phobos will be performed. The nominal instruments were selected, including the telescopic nadir imager for geomorphology (TENGOO) and optical radiometer composed of chromatic imagers (OROCHI). The scientific objective of TENGOO is to obtain the geomorphological features of Phobos and Deimos. The spatial resolution of TENGOO is $0.3 \mathrm{~m}$ at an altitude of $25 \mathrm{~km}$ in the quasi-satellite orbit. The scientific objective of OROCHI is to obtain material distribution using spectral mapping. $\mathrm{OROCHI}$ is composed of seven wide-angle bandpass imagers without a filter wheel and one monochromatic imager dedicated to the observation during the landing phase. Using these two instruments, we plan to select landing sites and obtain information that supports the analysis of return samples.

\section{Introduction}

Japan Aerospace Exploration Agency (JAXA)'s Martian Moons Exploration (MMX) is planned to be a sample return mission from Phobos, one of the satellites of Mars. Nominal instruments have been selected, including the telescopic nadir imager for geomorphology (TENGO0), optical radiometer composed of chromatic imagers (OROCHI), MMX infrared spectrometer (MIRS) [Barucci et al., this issue], Mars-moon exploration with gamma rays and neutrons (MEGANE) [Lawrence et al., 2019; Ernst et al., this issue], mass spectrum analyzer (MSA) [Yokota et al., this issue], circum-Martian dust monitor (CMDM) [Krueger et al., this issue], light detection and ranging (LIDAR) [Senshu et al., this issue] and Rover [Michel et al., this issue]. One of the scientific objectives of MMX is to determine the origin of the two Martian moons [Kuramoto et al., this issue]. Phobos and Deimos seem to be asteroids captured by the Mars' gravity, according to the results of a spectroscopic observation of the surface reflectance. Conversely, they also seem to have been formed by a large impact of a body with Mars and subsequent accretion. Elemental analysis is necessary to clarify the origin of the moons.

The MMX mission will acquire more than $10 \mathrm{~g}$ of regolith on the surface of Phobos [Usui et al., 2020; Fujiya et al., this issue]. A coring unit will be installed with a core diameter of $25 \mathrm{~mm}$. Assuming that the sample is representative of Phobos, we will be able to clarify the origin of the moons. To test this assumption, we should determine suitable landing sites and identify the uniformity or nonuniformity of the distribution of the surface material. Although we can obtain the globally averaged elemental composition using MEGANE, the distribution of the elemental composition cannot be obtained with a resolution of $25 \mathrm{~mm}$, comparable to the core diameter of the sample. $\mathrm{OROCHI}$ is a wide-angle multi-band imager, which enables the acquisition of spectral images of the moons. We can obtain global images from the quasi-satellite orbit and highest-resolution images during the landing operation. Additionally, we should select the landing site where the flatness is lower than $0.3 \mathrm{~m}$ in the diameter range of $5 \mathrm{~m}$ for safe landing of the MMX spacecraft. TENGOO is a telescopic camera, which enables to acquire highestresolution images from the quasi-satellite orbit for landing site selection. We also plan to observe the 
Mars climate from the orbit when we do not observe Phobos or Deimos [Ogohara et al., this issue]. In this paper, we describe the requirements for TENGOO and $\mathrm{OROCHI}$ and present our preliminary design.

\section{Performance Requirements}

One of the mission objectives of MMX is to spectroscopically reveal the surface-layer distribution of the materials that constitute Phobos with the spatial resolution required for the scientific evaluation of sampling points and geological structures, thereby constraining the Phobos' origin [Kuramoto et al., this issue]. To achieve this objective, the distributions of hydrous minerals and other related minerals should be obtained spectroscopically for the main parts of the full body in correspondence with its topography at horizontal spatial resolutions of below $20 \mathrm{~m}$. Global imaging operation will be performed when the MMX spacecraft will be in the quasi-satellite orbit (QSO); the typical altitude is $20 \mathrm{~km}$ in the QSO-low [Nakamura et al., this issue]. Thus, an angular resolution below $1 \mathrm{mrad}$ is required. We set the performance requirements for $\mathrm{OROCHI}$ as follows. The instantaneous field of view (iFoV) should be below $0.5 \mathrm{mrad} / \mathrm{pix}$. Seven bands were selected for spectroscopy. The center wavelengths are 390, 480, $550,650,730,860$, and $950 \mathrm{~nm}$, while the bandwidths are 50,30, 30, 40, 40, 40, and $60 \mathrm{~nm}$, respectively. The wavelengths of $390,480,550,860$, and $950 \mathrm{~nm}$ are the same as those of the ul-, b-, v-, $x-$, and p-bands of the optical navigation camera telescope (ONC-T) onboard the Hayabusa2 spacecraft [Kameda et al., 2017], which has seven bandpass filters. The wavelengths of 650 and $730 \mathrm{~nm}$ were selected to characterize the absorption around $650 \mathrm{~nm}$, specific to the red unit of Phobos [Genda et al., this issue; Fraeman et al., 2014]. The requirement for image quality was the modulation transfer functions (MTFs) of optics at Nyquist frequencies of above 0.3 for $480-860 \mathrm{~nm}$ and above 0.2 for 390 and $950 \mathrm{~nm}$. The signal-to-noise ratio (SNR) should be higher than 100 to detect the absorption around $650 \mathrm{~nm}$.

Material distributions should be obtained spectroscopically at a radius of $50 \mathrm{~m}$ or larger around the sampling point (at spatial resolutions of $1 \mathrm{~m}$ or better) [Kuramoto et al., this issue]. In the current operation plan, the MMX spacecraft will be right above the landing site at altitudes $<1 \mathrm{~km}$ in the descent phase [Nakamura et al., this issue]. Thus, the required angular resolution is the same as that above.

One or more images near the sampling point should be taken at the landing operation. The average distance between OROCHI and the surface of Phobos is designed to be $\sim 0.8 \mathrm{~m}$. The field of view (FoV) should be above 1 rad to obtain an image of the sampling point. The spatial resolution should be below 5 $\mathrm{mm}$ for multiband images, which corresponds to $1 / 5$ of the diameter of the sample core. In addition, the spatial resolution of monochromatic images should be below $1 \mathrm{~mm}$.

Images with resolutions below $0.3 \mathrm{~m}$ are required for landing site selection. To satisfy this requirement, we set the performance requirement for TENGOO as follows. The iFoV should be below $6 \mu \mathrm{rad} / \mathrm{pix}$, while the MTF of optics at Nyquist frequency should be above 0.2 , which is the lower limit to identify undulations at the size of above 2 pixels. If the iFoV is $6 \mu \mathrm{rad} / \mathrm{pix}, 2$ pixels correspond to $0.3 \mathrm{~m}$ at an altitude of $25 \mathrm{~km}$. Thus, landing site selection can be performed using the data obtained from the QSO. 


\section{Instrument Design}

\subsection{Design overview of TENGOO}

TENGOO has a camera and interface component, TENGOO-S, which is composed of optics, image sensor, analog front-end electronics (AFE), and analog-to-digital conversion (ADC) function, and TENGOO-E, which has a field-programmable gate array (FPGA), secondary power supply, and communication functions with the spacecraft system. TENGOO-S can operate when it is placed $2 \mathrm{~m}$ away from TENGOOE. Figures 3.1-1 and 3.1-2 show external views of TENGOO-S and TENGOO-E, respectively.

TENGOO-S has a catadioptric telescope. Its focal length is $947.8 \mathrm{~mm}$, while the effective F-number is 8.9 . The detector is an interline charge-coupled device (CCD), (KAI-08051). The pixel pitch of the CCD is 5.5 $\mu \mathrm{m}$, while the number of pixels is $3296 \times 2472$. Thus, the iFoV is $5.9 \mu \mathrm{rad}$, while the diagonal FoV is $1.34^{\circ}$. The optical system is panchromatic and consists of a primary mirror, secondary mirror, and corrective lens system consisting of four lenses.

The masses of TENGOO-S and $-E$ are 4.8 and $1.2 \mathrm{~kg}$, respectively. The dimensions of TENGOO-S and E are $\varphi 301.2 \mathrm{~mm} \times 352.5 \mathrm{~mm}$ and $201.2 \mathrm{~mm} \times 181.2 \mathrm{~mm} \times 49.5 \mathrm{~mm}$, respectively. The storage temperature is -30 to $+60^{\circ} \mathrm{C}$. The temperature of the telescope should be maintained at $+20 \pm 5^{\circ} \mathrm{C}$ during observation.

\subsection{Design overview of OROCHI}

$\mathrm{OROCHI}$ has a camera part and interface part in one housing. For multi-band observations, OROCHI has seven bandpass filters. The center wavelengths are $390,480,550,650,730,860$, and $950 \mathrm{~nm}$, while the bandwidths are $50,30,30,40,40,40$, and $60 \mathrm{~nm}$, respectively. OROCHI also has seven optics and seven CCD image sensors without filter wheel, although Hayabusa2/ONC-T has a filter wheel. The filter wheel has a limited number of rotations. If it stops in the middle of changing the filter, no further observations can be carried out. In addition, when the ground speed is high, particularly during the descent phase, the FoV moves while the filter is being changed. $\mathrm{OROCHI}$ has seven independent bandpass imagers to avoid these problems. In addition, $\mathrm{OROCHI}$ has a monochromatic camera $(550 \mathrm{~nm})$ dedicated to the observation during the landing phase. The spatial resolutions of the seven bandpass imagers are below 5 $\mathrm{mm}$ but above $1 \mathrm{~mm}$ because of the small distance $(\sim 0.8 \mathrm{~m})$. The focus of the monochromatic camera is adjusted for a distance of $0.8 \mathrm{~m}$ and its spatial resolution is $\sim 0.7 \mathrm{~mm}$. Thus, $\mathrm{OROCHI}$ has eight cameras in total. During the landing phase, the sample collection point may be in the shadow of the spacecraft. To prepare for such cases, OROCHI has a light emitting diode (LED) unit. Figures 3.2-1 and 3.2-2 show external views of $\mathrm{OROCHI}$ with and without hood, respectively

The OROCHI's eight optics are almost identical; however some gaps between lenses, aperture stop, etc., are optimized for each band. Its focal length is $13.23-13.57 \mathrm{~mm}$, and effective F-number is $5.8-6.4$. The detectors are interline CCDs (KAI-08051). The pixel pitch of the CCD is $5.5 \mu \mathrm{m}$ and the number of pixels is $3296 \times 2472$. Thus, the iFoV is $0.44-0.46$ milliradians, while the diagonal FoV is $83-85.5^{\circ}$. 
The mass of $\mathrm{OROCHI}$ is $12.13 \mathrm{~kg}$. Its dimensions are $243 \mathrm{~mm} \times 368 \mathrm{~mm} \times 368 \mathrm{~mm}$. The storage temperature is -30 to $+60^{\circ} \mathrm{C}$ and the operation temperature is -20 to $+55^{\circ} \mathrm{C}$.

\subsection{Detectors and electronics}

TENGOO and OROCHI use the same model number CCD, KAI-08051. This is an interline CCD. Its total number of pixels is $3364 \times 2520$, which includes dark reference pixels, and the number of active pixels is $3296 \times 2472$. We performed a comparative study between KAI-08051 and KAl-08052. KAl-08052 is a newer type of detector, which is approximately twice more sensitive in the near-infrared range, but has approximately three times higher dark current. During the landing phase, the temperature of the instrument is expected to increase, which increases the dark current, which is disadvantageous when LEDs are used to illuminate areas in the shadow of the spacecraft and acquire images with long exposure times. In order to ensure low development costs, we chose KAl-08051 for all CCD image sensors used in TENGOO and OROCHI.

Figures 3.3-1 and 3.3-2 show block diagrams of TENGOO and OROCHI, respectively. TENGOO has two AFEs for readout and uses two CCD output ports to increase the speed of the readout. The highest frame rate is 1.15 frame/s. TENGOO-E receives commands and sends out telemetry through SpaceWire (SpW). We use the high-speed serial interface Serializer Deserializer (SERDES) for the transmission of the imaging data. Both SpW and SERDES are redundant in systems $A$ and $B$. The power consumption of TENGOO is approximately $7 \mathrm{~W}$ in standby and approximately $15 \mathrm{~W}$ in imaging and readout.

OROCHI has almost the same electronics as TENGOO. It has only two AFEs for readout. Eight cameras are connected to the switch in parallel; therefore, it is not possible to read from more than two cameras simultaneously. For simultaneous imaging in multiple bands, $\mathrm{OROCHI}$ reads out up to seven wavelengths in sequence. Figure 3.3-3 shows the CCD readout timing diagram for simultaneous imaging at seven wavelengths with $\mathrm{OROCHI}$. As an illumination method for the landing phase, we use a combination of a white LED and multiple monochromatic LEDs. Figure 3.3-4 shows a conceptual diagram of the system of a white LED and five monochromatic LEDs (390, 480, 730, 860, and 950nm). Only the white LED is used at 550 and $650 \mathrm{~nm}$ because of its high intensity at these wavelengths. With this illumination unit, the area within a circle with a radius of $0.25 \mathrm{~m}$ becomes brighter than $1 / 100$ of the sunlit surface. The power consumption of $\mathrm{OROCH}$ is approximately $7 \mathrm{~W}$ in standby and $18 \mathrm{~W}$ in imaging and readout. The power consumption of the illumination unit is approximately $30 \mathrm{~W}$ in total.

\subsection{Optical design}

The optical system of TENGOO is catadioptric and consists of two mirrors (primary and secondary) and correction lens consisting of four lenses. An optical design schematic of TENGOO is shown in Figure 3.41. This system consists of rotationally symmetric aspheric primary and secondary mirrors and four spherical corrective lenses. The effective diameter of the primary mirror is $120 \mathrm{~mm}$, and the diameter of the shielded part is $50.5 \mathrm{~mm}$. In the order in which the incident light is reflected or transmitted, there is a primary mirror (G1), secondary mirror (G2), and corrective lenses (G3-6), leading to the sensor. The glass 
material is all fused silica for radiation tolerance. The length from the surface closest to the object to the sensor is $169.2 \mathrm{~mm}$ and the outer diameter of the primary mirror is $149.6 \mathrm{~mm}$.

The OROCHI optical system is a retrofocus type and consists of eight optical systems, seven for each observation wavelength and one for monochromatic imaging. An optical design schematic of the optics of OROCHI for a center wavelength of $550 \mathrm{~nm}$ is shown in Figure 3.4-2. The eight optical systems differ in the radius of curvature of the surface on the sensor side of G3, gap between $\mathrm{G} 2$ and $\mathrm{G} 3$, aperture diameter, and filter for each wavelength range and for monochromatic imaging. The spacing between G2 and G3 is adjusted for each wavelength range to reduce the effects of manufacturing errors. The differences in the optical systems for each wavelength and for monochromatic imaging are minor; structurally, they are almost identical. The outer diameter of G1 is $46 \mathrm{~mm}$. At the center wavelength of $550 \mathrm{~nm}$, the length from the first surface of the optical system to the sensor is $121.9 \mathrm{~mm}$.

As shown in Figure 3.4-2, the filter is placed between G2 and G3, to place the filter far away from the sensor to reduce stray light due to reflection between the filter and sensor and minimize the difference in the angle of incidence across the FoV to reduce the effect of the dependence of the filter on the angle of incidence. The back focus (distance from G6 to the sensor) should be long to reduce stray light due to reflection between $\mathrm{G} 6$ and the sensor. It is set to $24.9 \mathrm{~mm}$ at the center wavelength of $550 \mathrm{~nm}$.

\subsubsection{Radiometric performance}

The SNR required for TENGOO for topographic observation is $>30$. We calculated the surface brightness of Phobos under the following conditions:

1. The distance of Mars from the Sun is 1.666AU (aphelion).

2. The incidence angle of sunlight is $30^{\circ}$ and the emission angle is $0^{\circ}$.

3. Phobos has a Lambertian surface with a reflectance of $7 \%$.

The signal is calculated from the surface brightness, the optical system transmittance, and the quantum efficiency of the CCD. The random noise was calculated as the root sum square of statistical noise, readout noise, and dark current noise. The dark current will be corrected by subtracting the dark image from the observed image in operation. When Phobos and Mars are out of the FoV, multiple dark images are acquired in different viewing directions, and the portion without stars is used. Here, we assumed the CCD temperature is $+30^{\circ} \mathrm{C}$ for the highest dark current case. As a result, the exposure time should be longer than $1.2 \mathrm{~ms}$ for SNR $>30$. The ground speed at QSO is approximately $3 \mathrm{~m} / \mathrm{s}$, thus the shift of FoV in this exposure time $(36 \mathrm{~mm})$ is adequately smaller than the required resolution $(0.3 \mathrm{~m})$. The attitude stability of the spacecraft is $<3 \mu \mathrm{rad}(3 \sigma)$ for $10 \mathrm{~ms}$, which corresponds to $1 / 2$ pixel, thus the blurring of the image during this exposure time is also sufficiently smaller than the required resolution.

The SNR required for OROCHI for spectroscopic observation is $>100$. The F-numbers are 6.4 for 390-730 $\mathrm{nm}, 6.2$ for $860 \mathrm{~nm}$, and 5.8 for $950 \mathrm{~nm}$. As a result, the exposure times for SNR of 120 are $0.099 \mathrm{sec}$ for $390 \mathrm{~nm}, 0.071 \mathrm{sec}$ for $480 \mathrm{~nm}, 0.074 \mathrm{sec}$ for $550 \mathrm{~nm}, 0.086 \mathrm{sec}$ for $650 \mathrm{~nm}, 0.14 \mathrm{sec}$ for $730 \mathrm{~nm}, 0.49 \mathrm{sec}$ 
for $860 \mathrm{~nm}$, and $0.84 \mathrm{sec}$ for $950 \mathrm{~nm}$. The shift of FoV in this exposure time of $1 \mathrm{sec}$ is $\sim 3 \mathrm{~m}$, and it is adequately smaller than the resolution of $20 \mathrm{~m}$ required for QSO observation. The attitude stability of the spacecraft is $<0.17 \mathrm{mrad}(3 \sigma)$ for $1 \mathrm{~s}$, which corresponds to 0.4 pixels, thus the blurring of the image during the shorter exposure time is also sufficiently smaller than the required resolution.

We need longer exposure time when we obtain the image of the surface in the shadow of spacecraft using the LED illumination because the surface brightness is $1 / 100$ of the sunlit surface. To achieve SNR $>100$, we have to use $2 \times 2$ binning mode and the required exposure times are $4.84 \mathrm{sec}, 3.56 \mathrm{sec}, 3.64$ $\mathrm{sec}, 4.39 \mathrm{sec}, 7.14 \mathrm{sec}, 26.7 \mathrm{sec}$, and $52.4 \mathrm{sec}$, respectively. In addition, we have to take multiple images with shorter exposure time for $860 \mathrm{~nm}$ and $950 \mathrm{~nm}$ because the number of integrated electrons exceed the full well capacity of CCD.

\subsubsection{Optical resolution}

The MTFs of TENGOO were calculated in the spectral range of $350-950 \mathrm{~nm}$. Here we have considered the spectrum of incident flux, quantum efficiency of the detector, and optics transmittance. The result is shown in Figure 3.4.1-1. The MTF at Nyquist frequency $(91 \mathrm{lp} / \mathrm{mm})$ is more than 0.29 in design. Although invar, a material with low thermal expansion, is used in the support structure of the telescope, the temperature of the telescope needs to be controlled within a range of $+/-5^{\circ} \mathrm{C}$. Figure 3.4.1-2 shows the MTF at Nyquist frequency is 0.25 at $-5^{\circ} \mathrm{C}$ relative to the nominal temperature and the guaranteed smallest value of MTF is 0.2. Note that the change in MTF when the pressure changes from ambient to vacuum is negligible because the refractive power is mainly carried by the mirror.

The MTF of OROCHI was calculated by assigning the same weight to the three wavelengths of the central, longest, and shortest wavelengths for each band (e.g., for a central wavelength of $390 \mathrm{~nm}$ and a bandwidth of $50 \mathrm{~nm}$, the same weight was assigned to the three wavelengths of $415 \mathrm{~nm}, 390 \mathrm{~nm}$, and $365 \mathrm{~nm}$ ). The calculated MTFs for each band are shown in Figures 3.4.2-3 to 3.4.2-9. The nominal values of MTFs at the Nyquist frequency $(91 \mathrm{lp} / \mathrm{mm})$ are $0.31-0.44$ at a distance of $20 \mathrm{~km}$ over the entire FoV. As shown in Figure 3.4.2-10, the MTF at the Nyquist frequency is 0.31 over the entire FoV for monochromatic imaging during the landing phase at a distance of $0.8 \mathrm{~m}$ with a center wavelength of $550 \mathrm{~nm}$ and a bandwidth of $30 \mathrm{~nm}$.

\section{Summary}

The scientific objectives of TENGOO and OROCHI are to obtain the geomorphological features and material distribution using spectral mapping of Phobos and Deimos. Images with resolutions below 0.3 $\mathrm{m}$ are required for landing site selection. To satisfy this requirement, the performance requirements for TENGOO are iFoV $<6 \mu \mathrm{rad} /$ pix, MTF at Nyquist frequency $>0.2$, and SNR $>30$. OROCHI should have seven bandpass imagers and the performance requirements are iFoV $<0.5 \mathrm{mrad} / \mathrm{pix}$, MTF at Nyquist frequency $>0.2$, and SNR $>100$. In the preliminary design, TENGOO has a camera and interface component, and the optical system of TENGOO is catadioptric. Its iFoV of TENGOO is $5.9 \mu \mathrm{rad}$ and MTF at Nyquist frequency is 0.29 in design. The temperature of the telescope should be maintained at $+20 \pm 5$ 
${ }^{\circ} \mathrm{C}$ during observation to achieve MTF at Nyquist frequency $>0.2$. $\mathrm{OROCHI}$ has a camera part and interface part in one housing. The camera part is composed of seven optics, seven bandpass filters, and seven CCD image sensors for multi-band imaging. Besides, $\mathrm{OROCHI}$ has a monochromatic camera for observation during the landing phase. The iFoVs are $0.44-0.46$, MTF at Nyquist frequency is above 0.3, and SNR is $>100$ in nominal operation. We need longer exposure time when we obtain the image of the surface in the shadow of the spacecraft using the LED illumination because the surface brightness is $1 / 100$ of the sunlit surface. we have to use $2 \times 2$ binning mode and the required exposure times to achieve SNR > 100.

\section{Abbreviations}

ADC: Analog to Digital Conversion

AFE: Analog Front-end Electronics

CCD: Charge Coupled Device

CMDM: Circum-Martian Dust Monitor

FoV: Field of View

iFoV: instantaneous Field of View

FPGA: Field-Programmable Gate Array

JAXA: Japan Aerospace Exploration Agency

LED: Light Emitting Diode

LIDAR: Llght Detection And Ranging

MEGANE: Mars-moon Exploration with GAmma rays and Neutrons

MIRS: MMX-InfRared Spectrometer

MMX: Martian Moon eXplorer

MSA: Mass Spectrum Analyzer

MTF: Modulation Transfer Function

ONC-T: Optical Navigation Camera Telescope

OROCHI : Optical RadiOmeter composed of CHromatic Imagers

QSO: Quasi Satellite Orbit 
SERDES: SERializer DESerializer

SNR: Signal to Noise Ratio

SpW: SpaceWire

TENGOO: Telescopic Nadir Imager for GeOmOrphology

\section{Declarations}

\section{Availability of data and materials}

\section{Competing interests}

The authors declare that they have no competing interest

\section{Funding}

TENGOO and OROCHI instruments are financed by JAXA.

This study is supported by the following grants: Japan Society for the Promotion of Science (JSPS) KAKENHI 17KK0097; $19 \mathrm{H} 00727$

\section{Author's contributions}

SK contributed to the design and development of the instrument and writing.

MO, HK, CLK, YG, HM, SF, TA, KH, SM, NT, TT, KI, TO, TI, PH, RI contributed to the design of electronics, selection of detector, structural and thermal design.

KE, RF, NO, KM, MM, KT, HS contributed to the optical design.

All others provided feedback.

\section{Acknowledgements}

This study is supported by the following grants: Japan Society for the Promotion of Science (JSPS) KAKENHI 17KK0097; 19H00727. We thank Ms. Shiho Kobayashi (Tohoku University), Dr. Isao Yoda (Laboratory for Advanced Nuclear Energy, TiTech), Dr. Kazuo Tanaka (Cyclotron and Radioisotope Center (CYRIC), Tohoku University), and JAXA MMX system team member for their support.

\section{References}

1. Barucci et al., this issue, MIRS an Imaging spectrometer for the MMX mission. 
2. Ernst et al., this issue. Use of the Small Body Mapping Tool for the MMX MEGANE Investigation.

3. Fraeman et al., 2014, Spectral absorptions on Phobos and Deimos in the visible/near infrared wavelengths and their compositional constraints. Icarus, 229, 196-205.

4. Fujiya et al., this issue, Analytical protocols for Phobos regolith samples returned by the Martian Moons eXploration (MMX) mission.

5. Genda et al., this issue, Origin of Martian moons and solar system evolution in the MMX mission.

6. Kameda et al., 2017, Preflight Calibration Test Results for Optical Navigation Camera Telescope (ONC-T) Onboard the Hayabusa2 Spacecraft. Space Science Reviews, 208, 17-31.

7. Krueger et al., this issue, Modelling cometary meteoroid trail traverses of the Martian Moons eXploration (MMX) spacecraft en route to Phobos.

8. Kuramoto et al., this issue, Martian Moons Exploration MMX: Sample Return Mission to Phobos Elucidating Formation Processes of Habitable Planets.

9. Lawrence et al., 2019, Measuring the Elemental Composition of Phobos: The Mars-moon Exploration with GAmma rays and NEutrons (MEGANE) Investigation for the Martian Moons eXploration (MMX) Mission. Earth and Space Science, 6, 2605-2623.

10. Michel et al., this issue. Performing in-situ surface investigations on Phobos.

11. Nakamura et al., this issue, Scientific observation plan of Phobos and Deimos from MMX spacecraft.

12. Ogohara et al., this issue, The Mars system revealed by the Martian Moons eXploration mission.

13. Senshu et al., this issue, Development of LIDAR onboard MMX.

14. Usui et al., 2020, The Importance of Phobos Sample Return for Understanding the Mars-Moon System. Space Science Reviews, 216, 49

15. Yokota et al., this issue, In situ observations of ions and magnetic field around Phobos: The Mass Spectrum Analyzer (MSA) for the Martian Moons eXploration (MMX) mission.

\section{Figures}



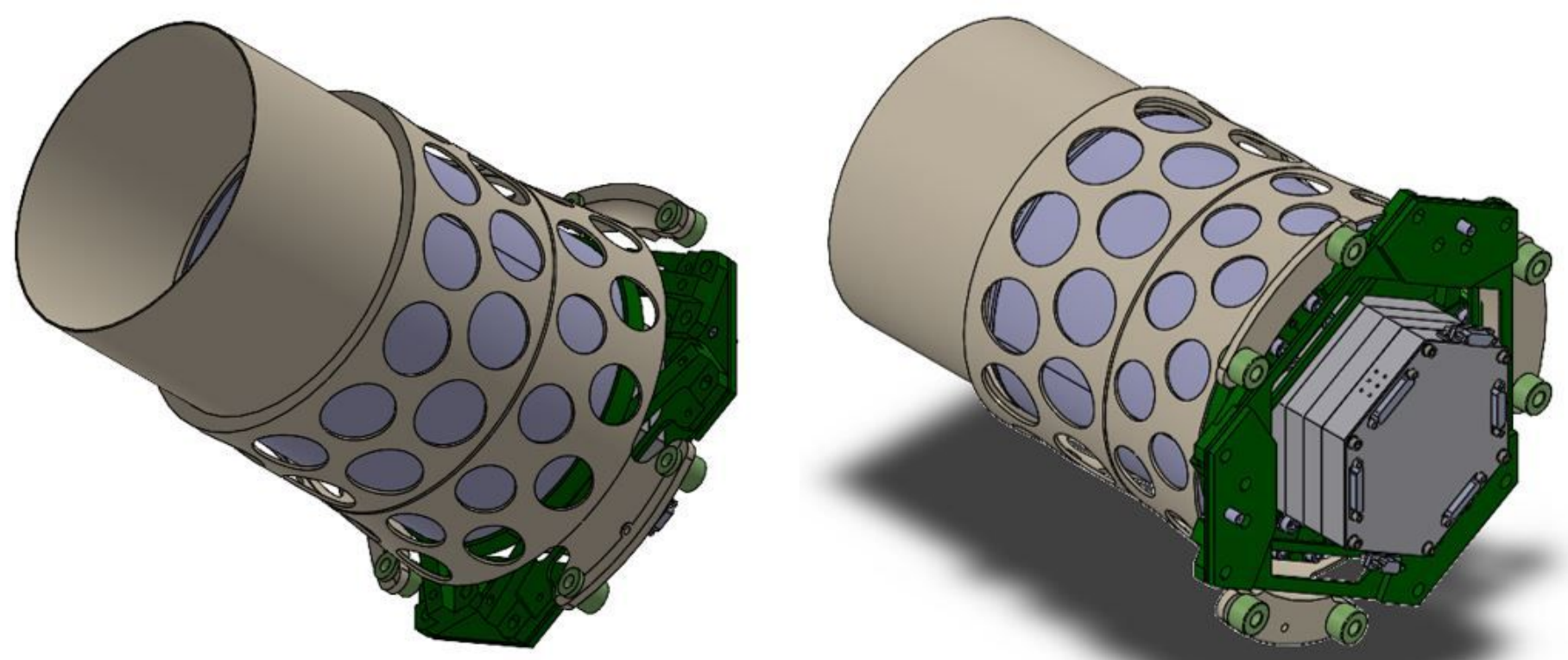

\section{Figure 1}

External views of TENGOO-S
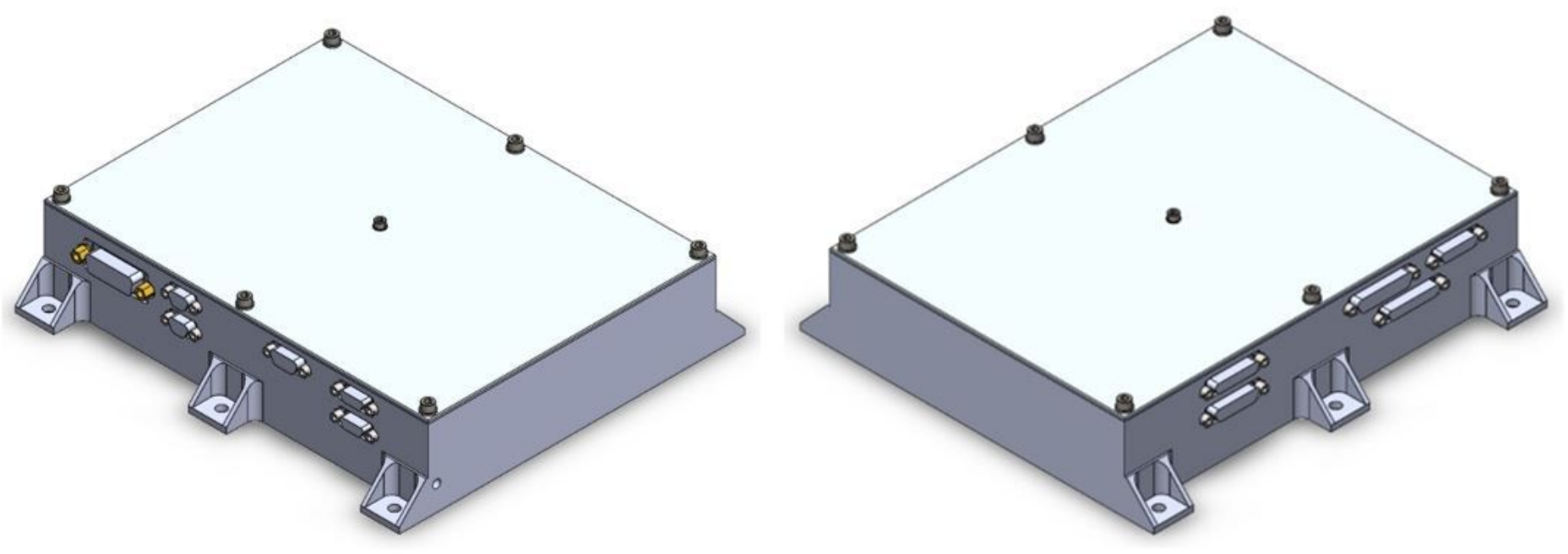

Figure 2

External views of TENGOO-E 


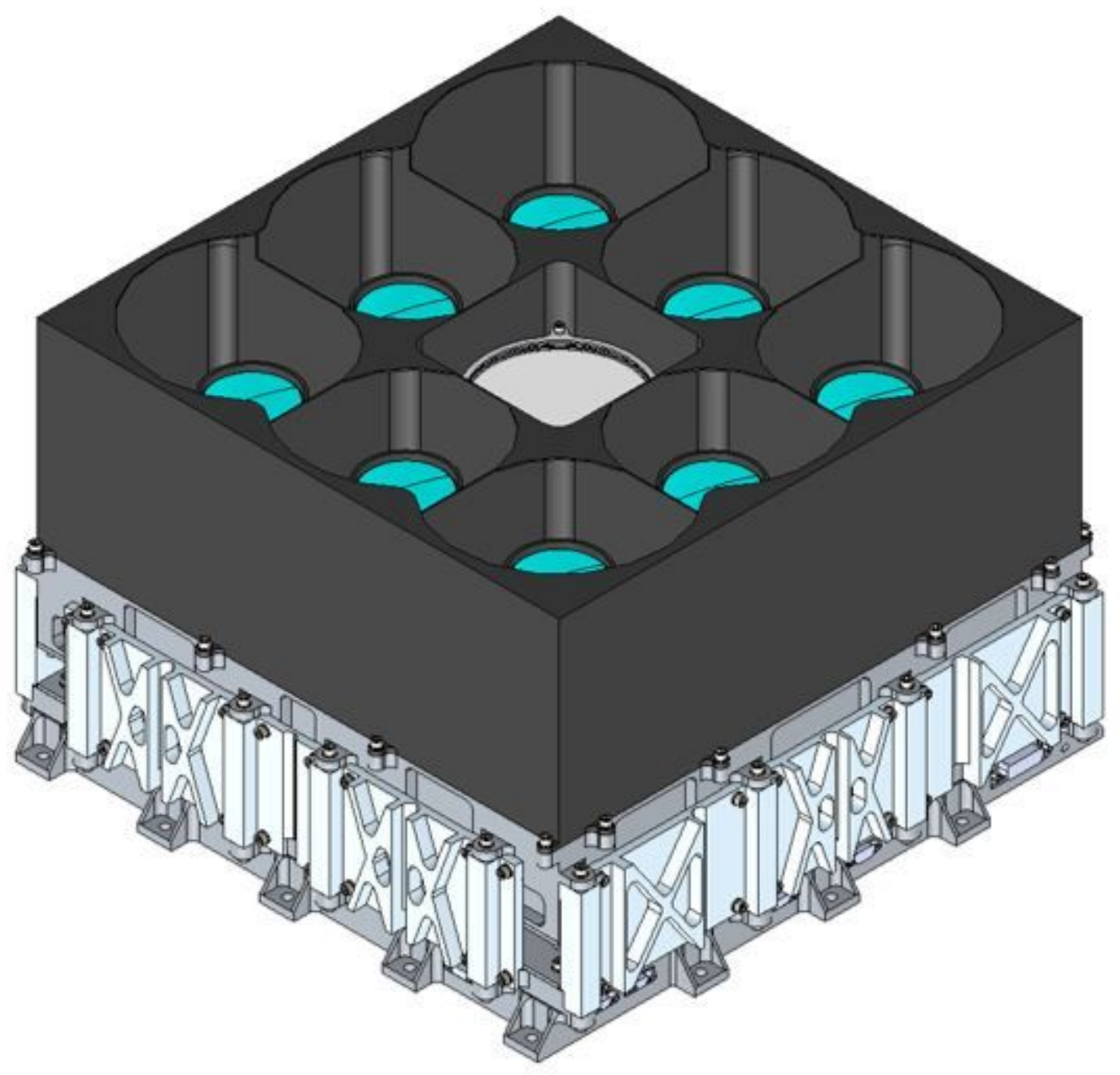

Figure 3

External view of OROCHI 


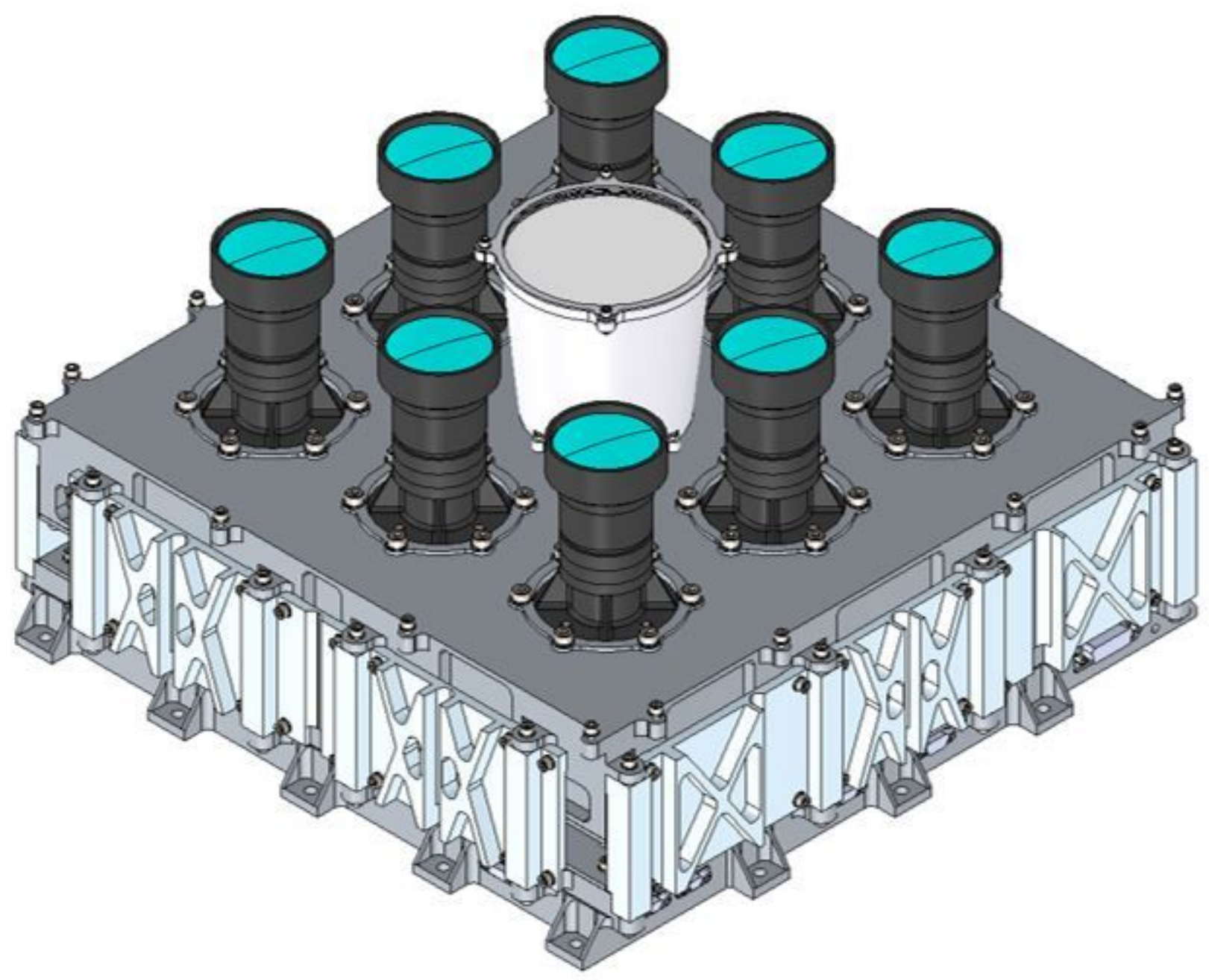

Figure 4

External view of $\mathrm{OROCH}$ without hood 


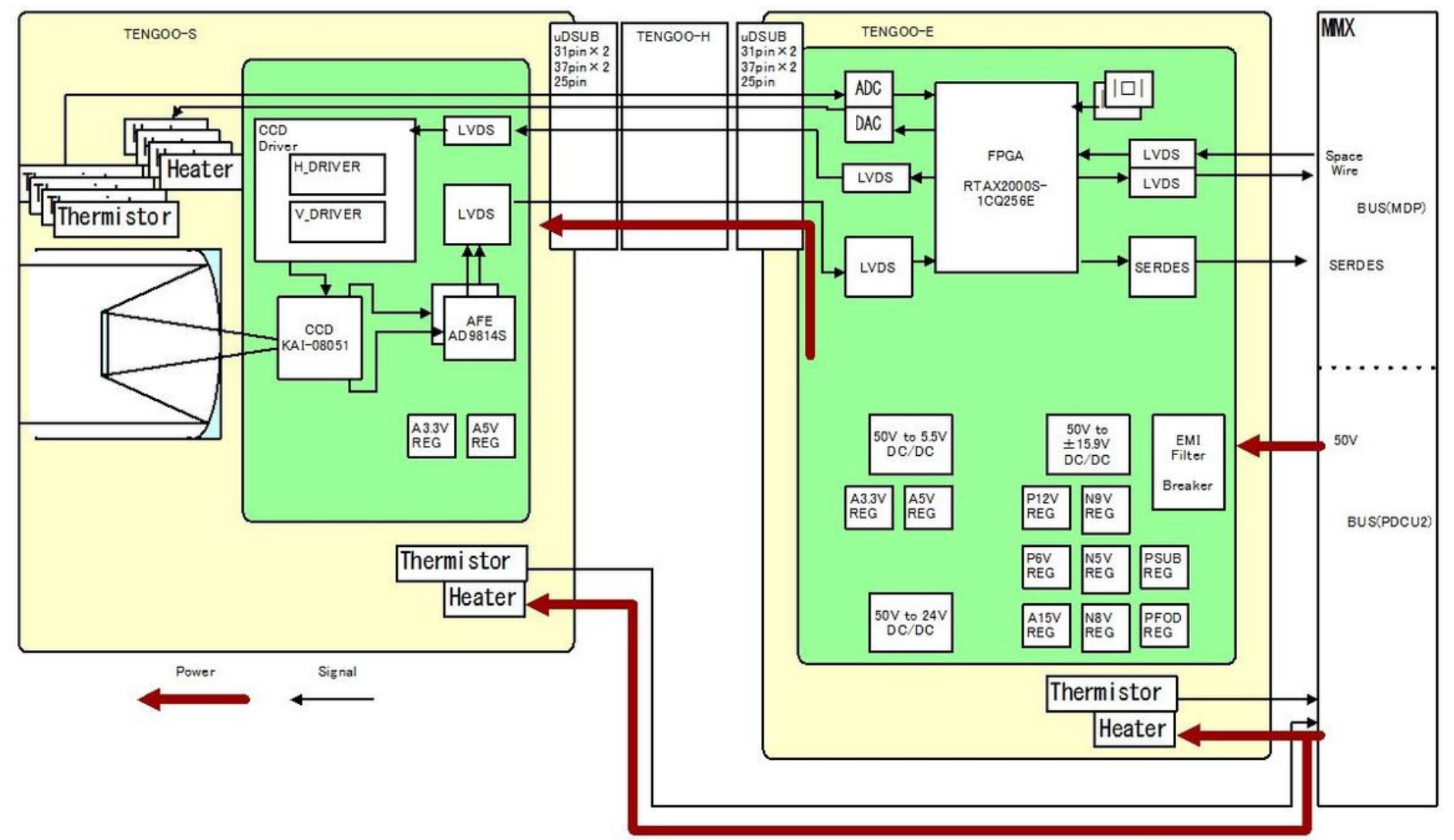

Figure 5

TENGOO block diagram

Figure 6

OROCHI block diagram

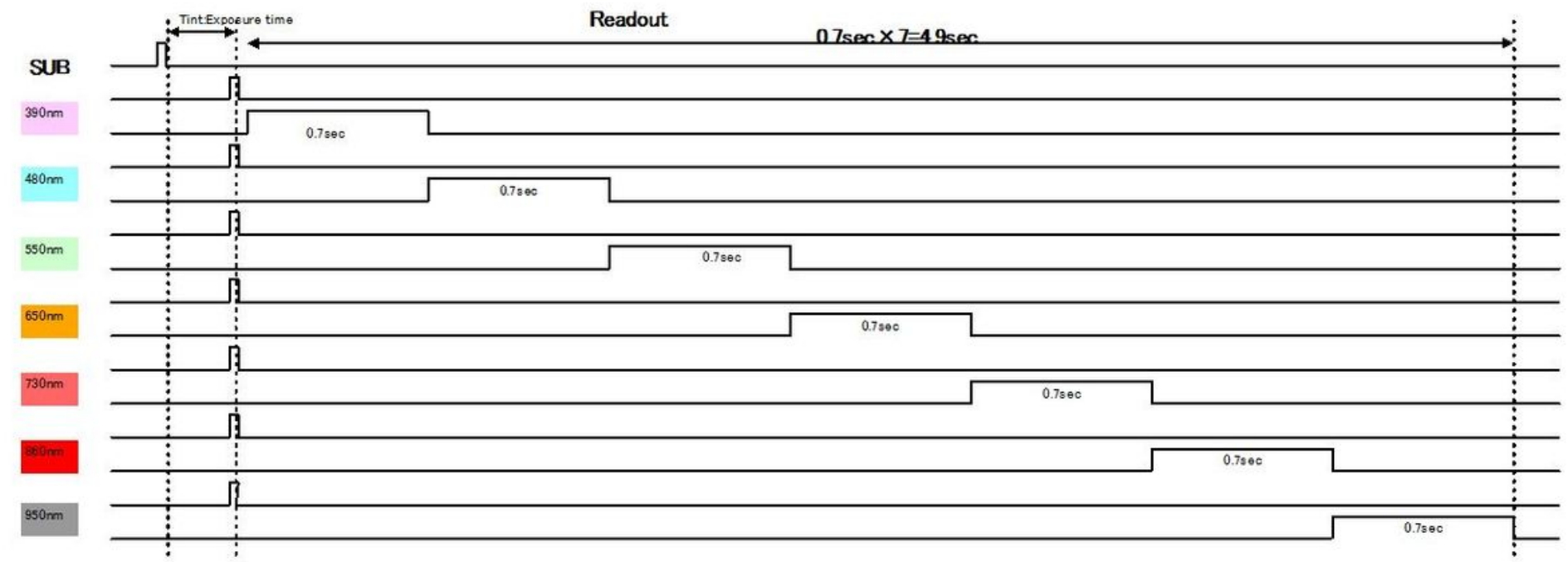

Figure 7 
$\mathrm{OROCHI}$ readout timing diagram for simultaneous seven-band imaging

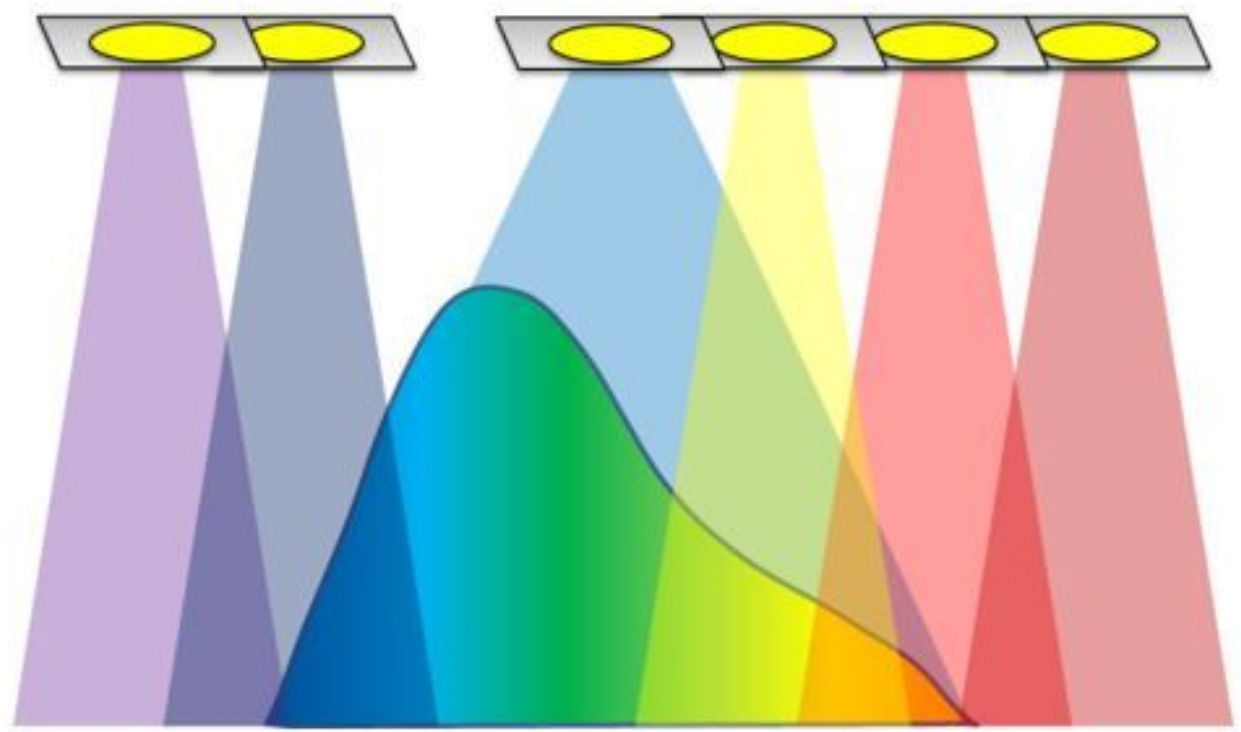

Figure 8

Conceptual diagram of the system of a white LED and five monochromatic LEDs $(390,480,730,860$, and 950nm) 


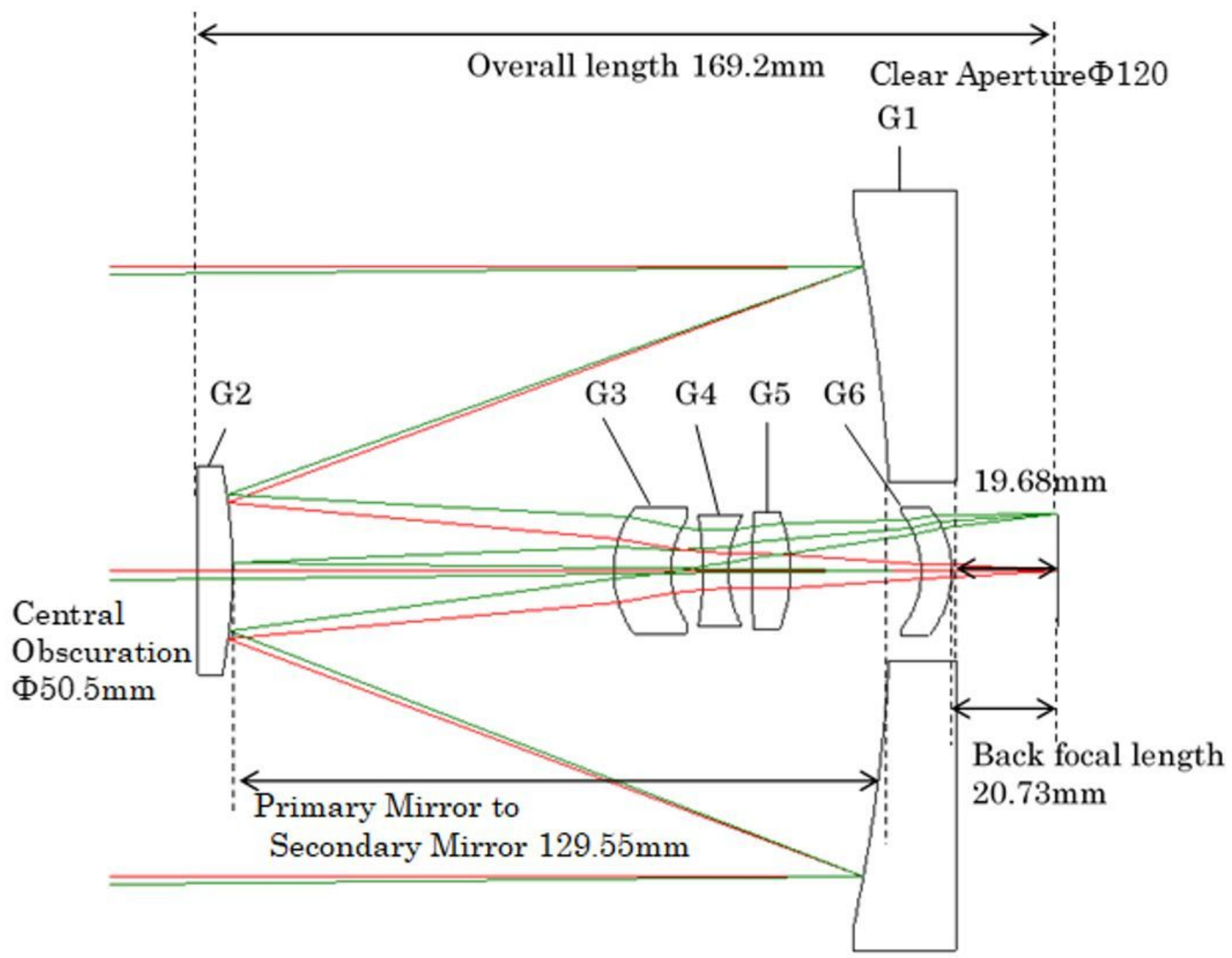

Figure 9

TENGOO optical design schematic 


\section{Overall Length $121.3 \sim 122.8 \mathrm{~mm}$}

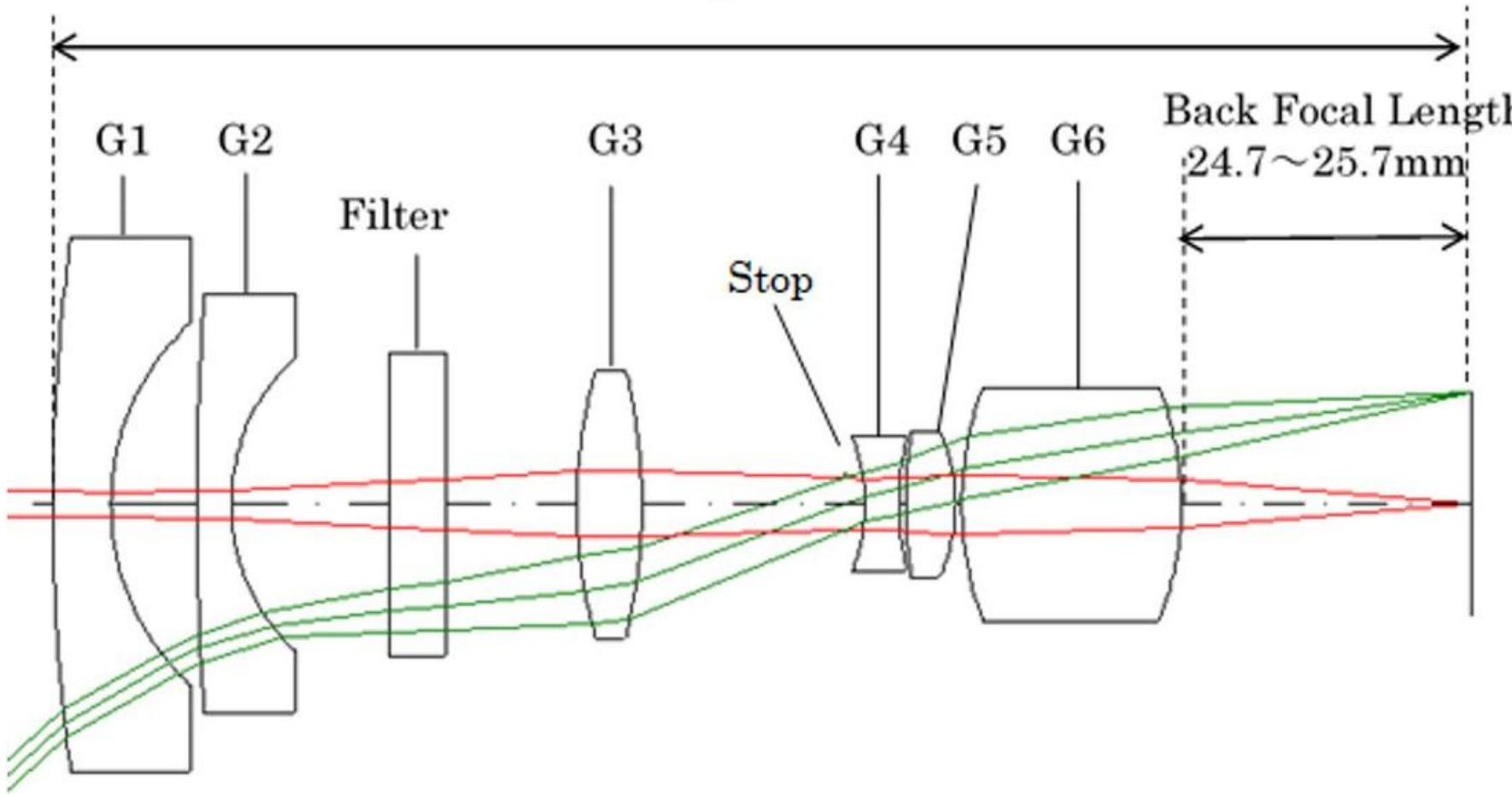

Figure 10

OROCHI optical design schematic

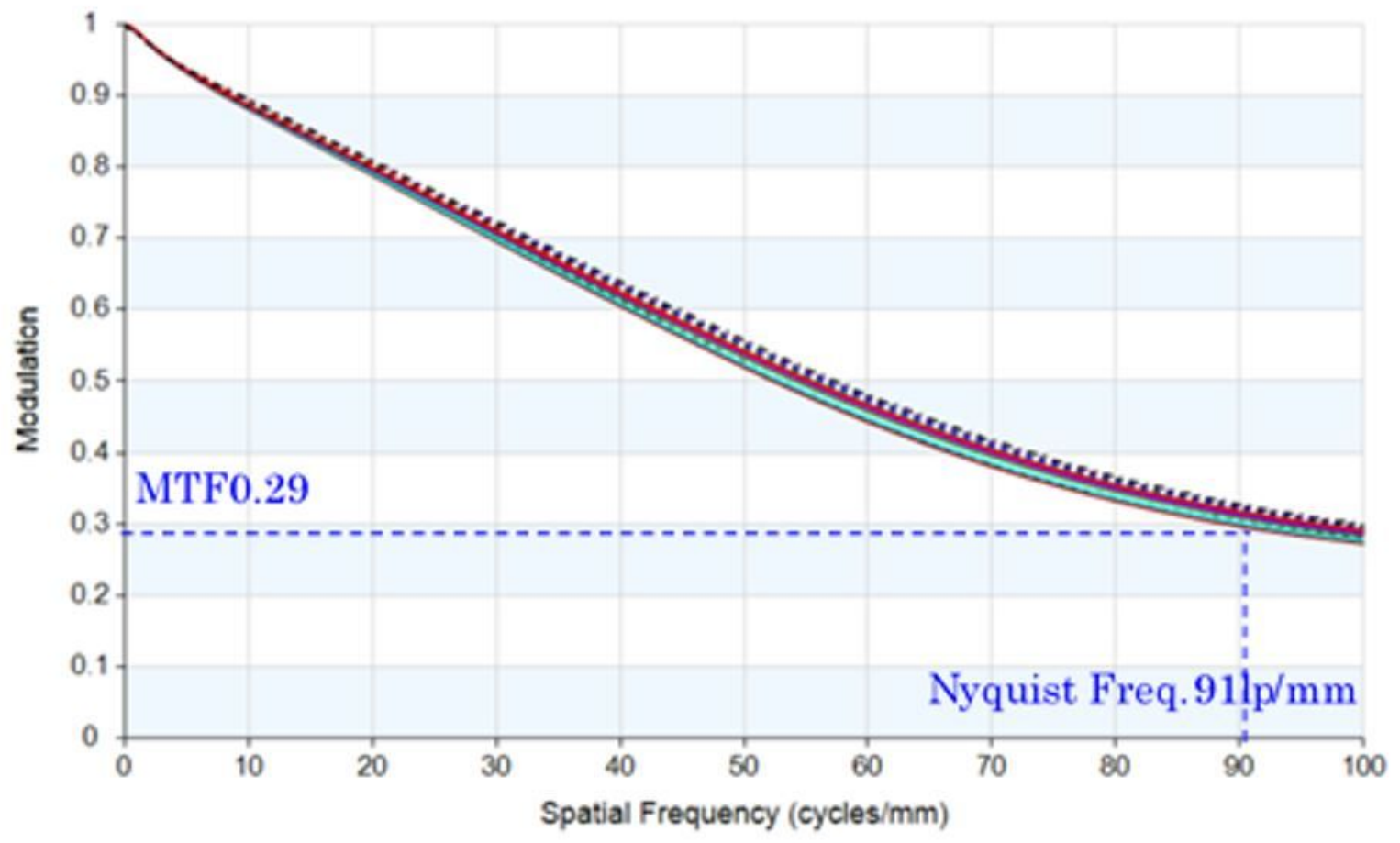

-F1: Y Diff. Limit

-F1: X Diff. Limit

- F1: $Y$ (RIH) $0.000 \mathrm{~mm}$

- F1: X (RIH) $0.000 \mathrm{~mm}$

- F2: Y (RIH) $2.833 \mathrm{~mm}$

- F2: X (RIH) $2.833 \mathrm{~mm}$

- F3: Y (RIH) $5.665 \mathrm{~mm}$

* F3: X (RIH) $5.665 \mathrm{~mm}$

- F4: Y (RIH) $6.798 \mathrm{~mm}$

. F4: X (RIH) $6.798 \mathrm{~mm}$

- F5: $Y(R I H) 7.931 \mathrm{~mm}$

- F5: X (RIH) $7.931 \mathrm{~mm}$

- F6: Y (RIH) $9.615 \mathrm{~mm}$

-F6: $X(R I H) 9.615 \mathrm{~mm}$

- F7: $Y(R I H) 11.330 \mathrm{~mm}$

. F7: X (RIH) $11.330 \mathrm{~mm}$

Figure 11

TENGOO optics MTF 


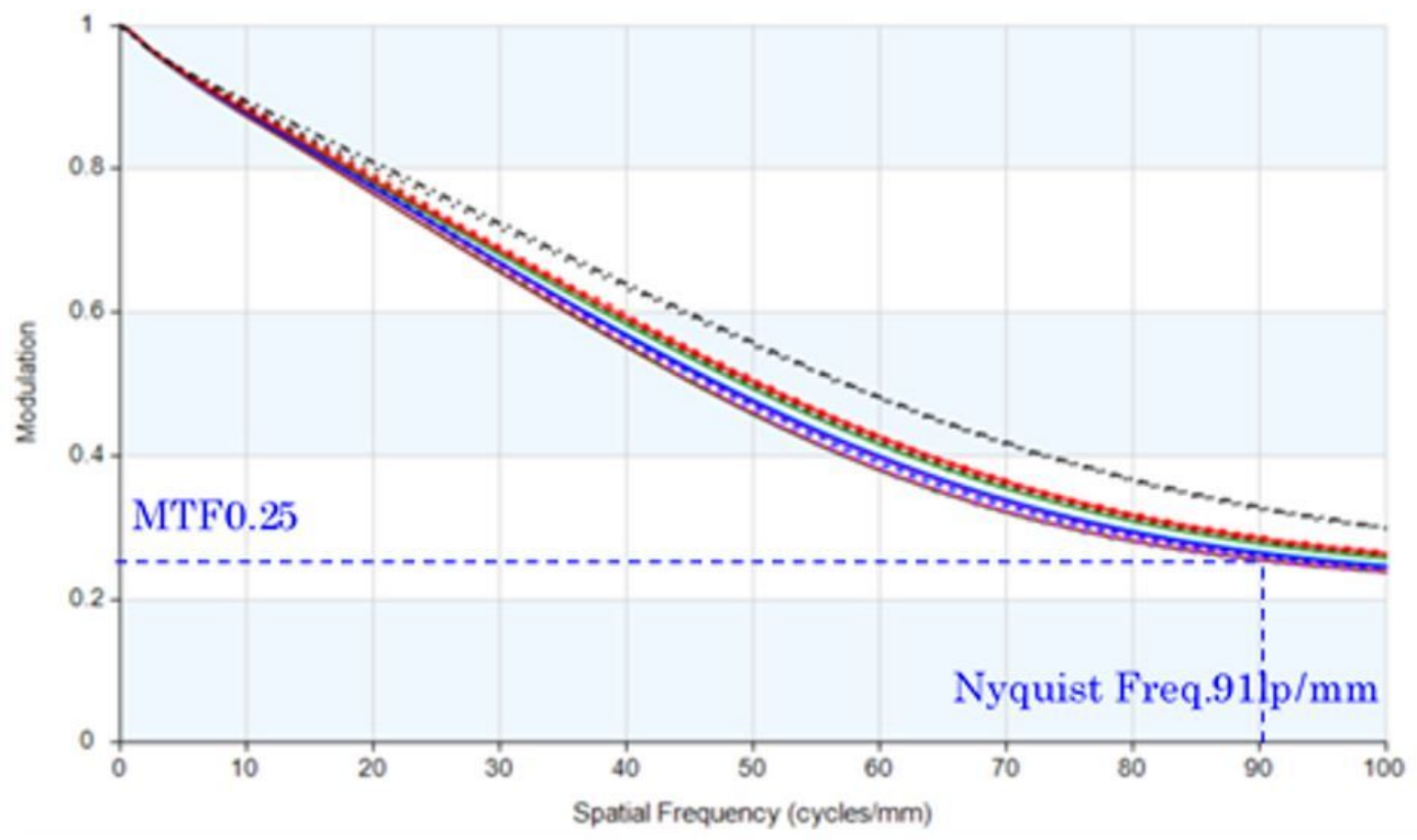

.. F1: Y Diff. Limit

-.F1: X Diff. Limit

- F1: Y (RIH) $0.000 \mathrm{~mm}$

-. F1: $X(R \mid H) 0.000 \mathrm{~mm}$

- F2: $Y($ RIH $) 2833 \mathrm{~mm}$

-F F X (RIH) $2833 \mathrm{~mm}$

- F3: $Y(R I H) 5.665 \mathrm{~mm}$

.. F3: X(RIH) $5.665 \mathrm{~mm}$

- F4: Y (RIH) $6.798 \mathrm{~mm}$

.. F4: X $(R I H) 6.798 \mathrm{~mm}$

- FS: Y (RIH) $7.931 \mathrm{~mm}$

-. F5: X (RIH) $7.931 \mathrm{~mm}$

- F6: $Y(R I H) 9.615 \mathrm{~mm}$

F6: X (RIH) $9.615 \mathrm{~mm}$

-F7: $Y(R I H) 11.330 \mathrm{~mm}$

. F7: X(RIH) $11.330 \mathrm{~mm}$

Figure 12

TENGOO optics MTF at $-5^{\circ} \mathrm{C}$ relative to the nominal temperature.

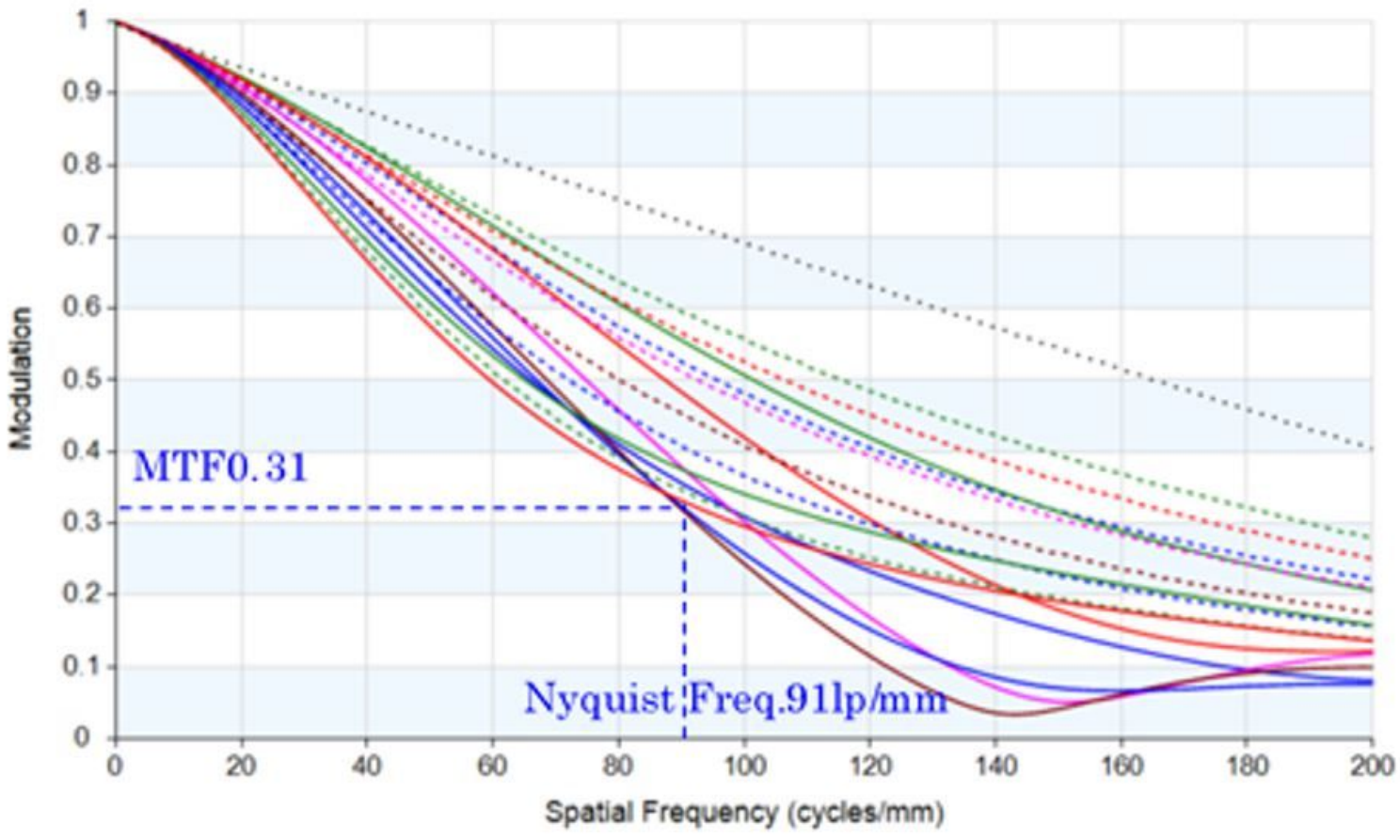

-F1: Diff. Limit

- F1: (RIH) $0.000 \mathrm{~mm}$

- F2: T (RIH) $2.404 \mathrm{~mm}$

- F2. R (RIH) $2.404 \mathrm{~mm}$

- F3: T (RIH) $4.808 \mathrm{~mm}$

. F3: R (RIH) $4.808 \mathrm{~mm}$

- F4: T (RIH) $5.769 \mathrm{~mm}$

- F4: R (RIH) $5.769 \mathrm{~mm}$

- F5: T (RIH) $6.798 \mathrm{~mm}$

* F5: R (RIH) $6.798 \mathrm{~mm}$

- F6: T (RIH) $7.692 \mathrm{~mm}$

* F6: R (RIH) $7.692 \mathrm{~mm}$

- F7: T (RIH) $8.654 \mathrm{~mm}$

. F7: R (RIH) $8.654 \mathrm{~mm}$

- F8: T (RIH) $9.615 \mathrm{~mm}$

.. F8: R (RIH) $9.615 \mathrm{~mm}$

\section{Figure 13}

MTF vs Spatial Freq. Design Nominal at Objective Distance 20km (Central Wavelength 390nm, Bandwidth 50nm) 


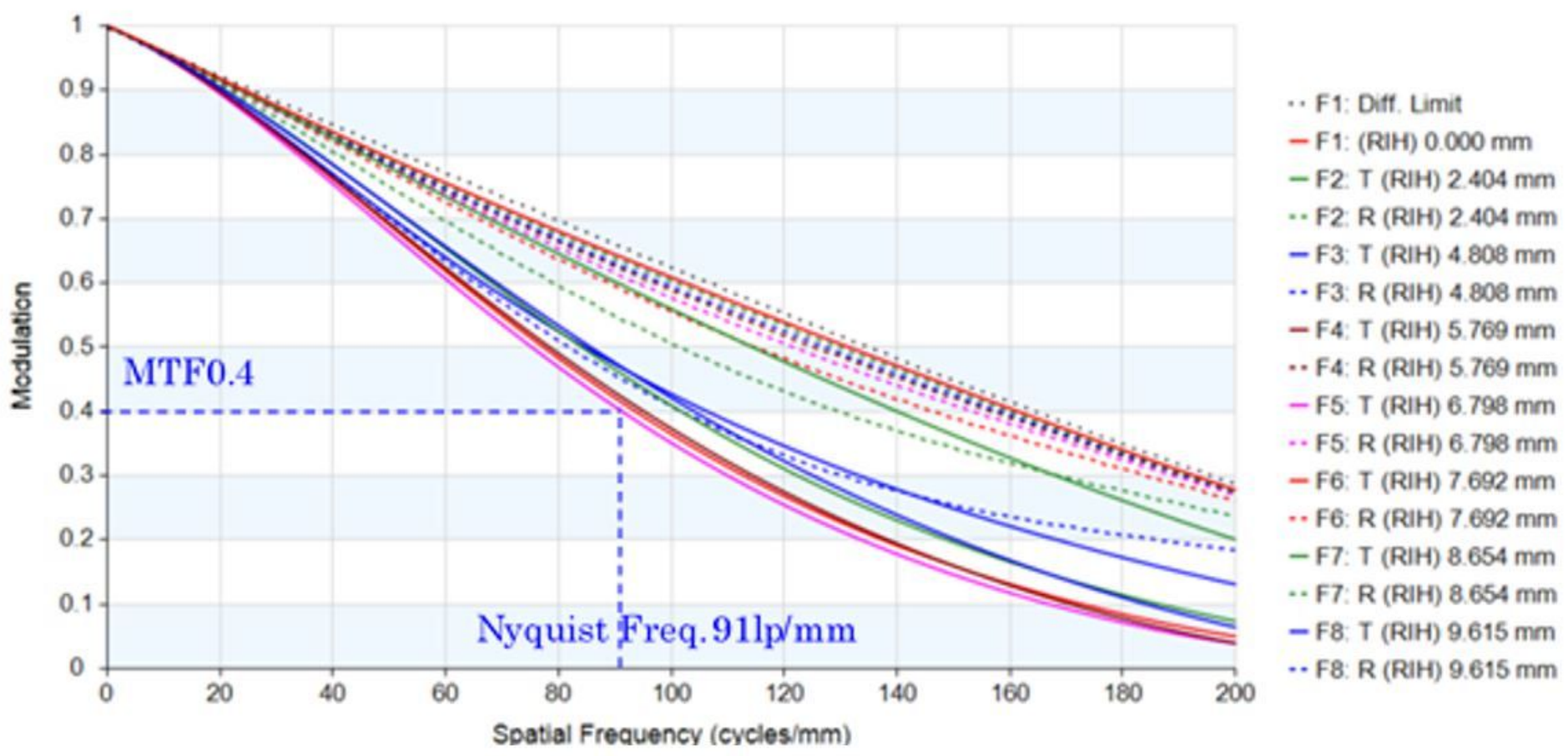

Figure 14

MTF vs Spatial Freq. Design Nominal at Objective Distance 20km (Central Wavelength 480nm, Bandwidth 30nm)

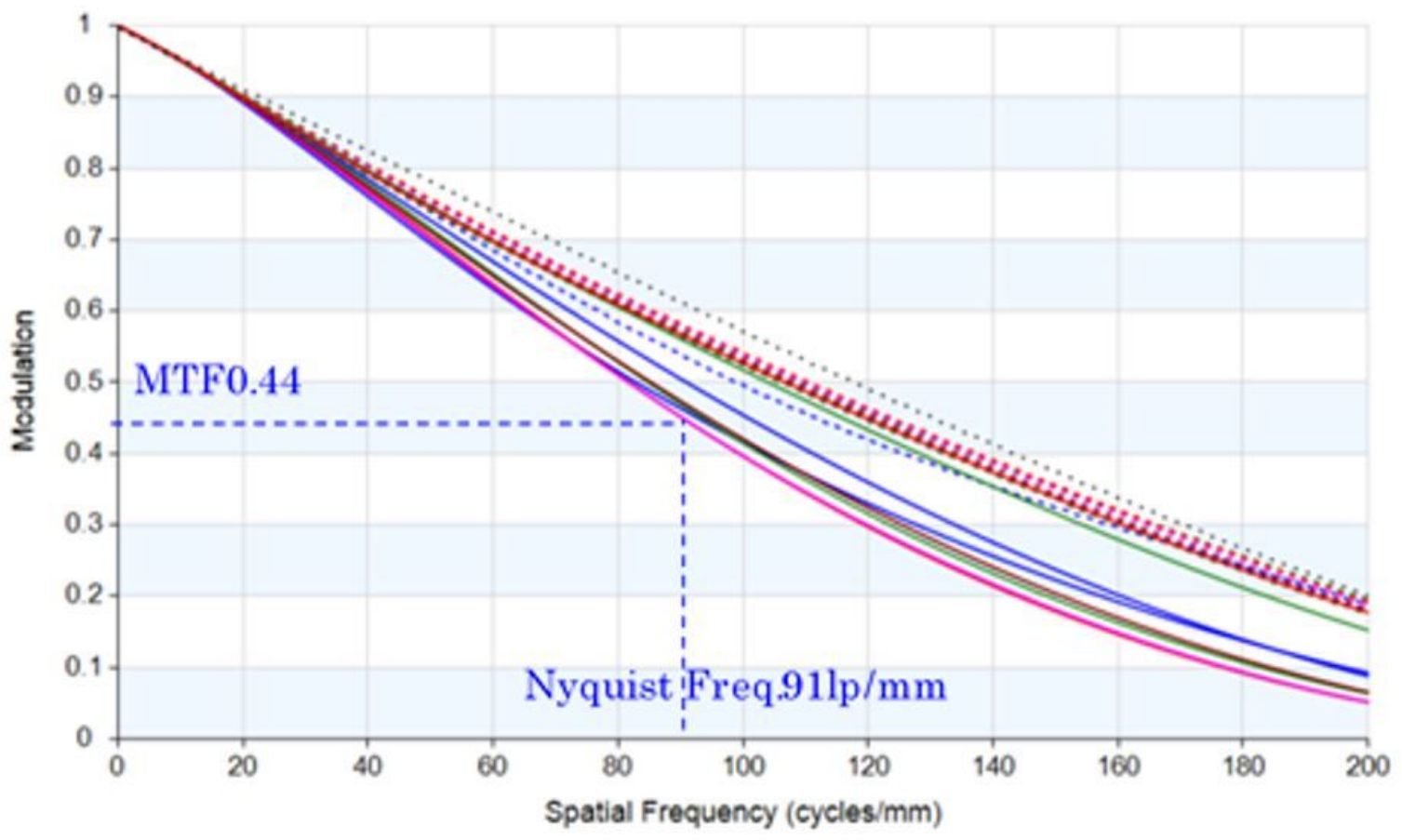

* F1: Diff. Limit

$-F 1:$ (RIH) $0.000 \mathrm{~mm}$

- F2: T (RIH) $2.404 \mathrm{~mm}$

* F2: R (RIH) $2404 \mathrm{~mm}$

- F3: T (RIH) $4.808 \mathrm{~mm}$

- F3: R (RIH) $4.808 \mathrm{~mm}$

- F4: T (RIH) $5.769 \mathrm{~mm}$

“ F4: R (RIH) $5.769 \mathrm{~mm}$

- F5: T (RIH) $6.798 \mathrm{~mm}$

. F5: R (RIH) $6.798 \mathrm{~mm}$

- F6: T (RIH) $7.692 \mathrm{~mm}$

- F6: R (RIH) $7.692 \mathrm{~mm}$

- F7: T (RIH) $8.654 \mathrm{~mm}$

. F7: R (RIH) $8.654 \mathrm{~mm}$

- F8: T (RIH) $9.615 \mathrm{~mm}$

- F8: R (RIH) $9.615 \mathrm{~mm}$

\section{Figure 15}

MTF vs Spatial Freq. Design Nominal at Objective Distance 20km (Central Wavelength 550nm, Bandwidth 30nm) 


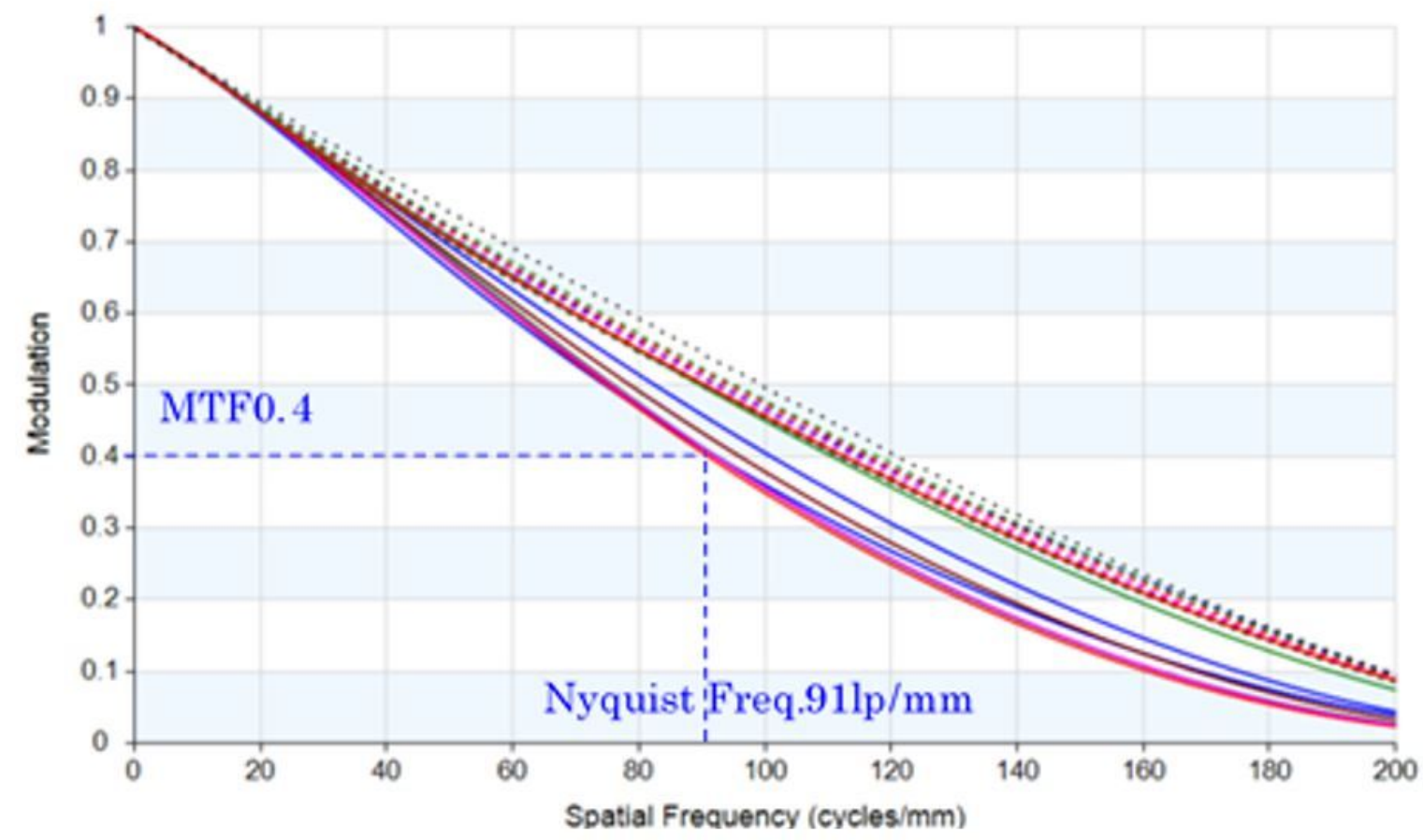

. F1: Diff. Limit

- F1: (RIH) $0.000 \mathrm{~mm}$

- F2: T (RIH) $2.404 \mathrm{~mm}$

.- F2: R (RIH) $2.404 \mathrm{~mm}$

-F3: T (RIH) $4.808 \mathrm{~mm}$

-. F3: R (RIH) $4.808 \mathrm{~mm}$

- F4: T (RIH) $5.769 \mathrm{~mm}$

- F4: R (RIH) $5.769 \mathrm{~mm}$

- F5: T (RIH) $6.798 \mathrm{~mm}$

. F5: R (RIH) $6.798 \mathrm{~mm}$

- F6: T (RIH) $7.692 \mathrm{~mm}$

. F6: R (RIH) $7.692 \mathrm{~mm}$

- F7: T (RIH) $8.654 \mathrm{~mm}$

- F7: R (RIH) $8.654 \mathrm{~mm}$

- F8: T (RIH) $9.615 \mathrm{~mm}$

- F8: R (RlH) $9.615 \mathrm{~mm}$

\section{Figure 16}

MTF vs Spatial Freq. Design Nominal at Objective Distance 20km (Central Wavelength 650nm, Bandwidth 40nm)

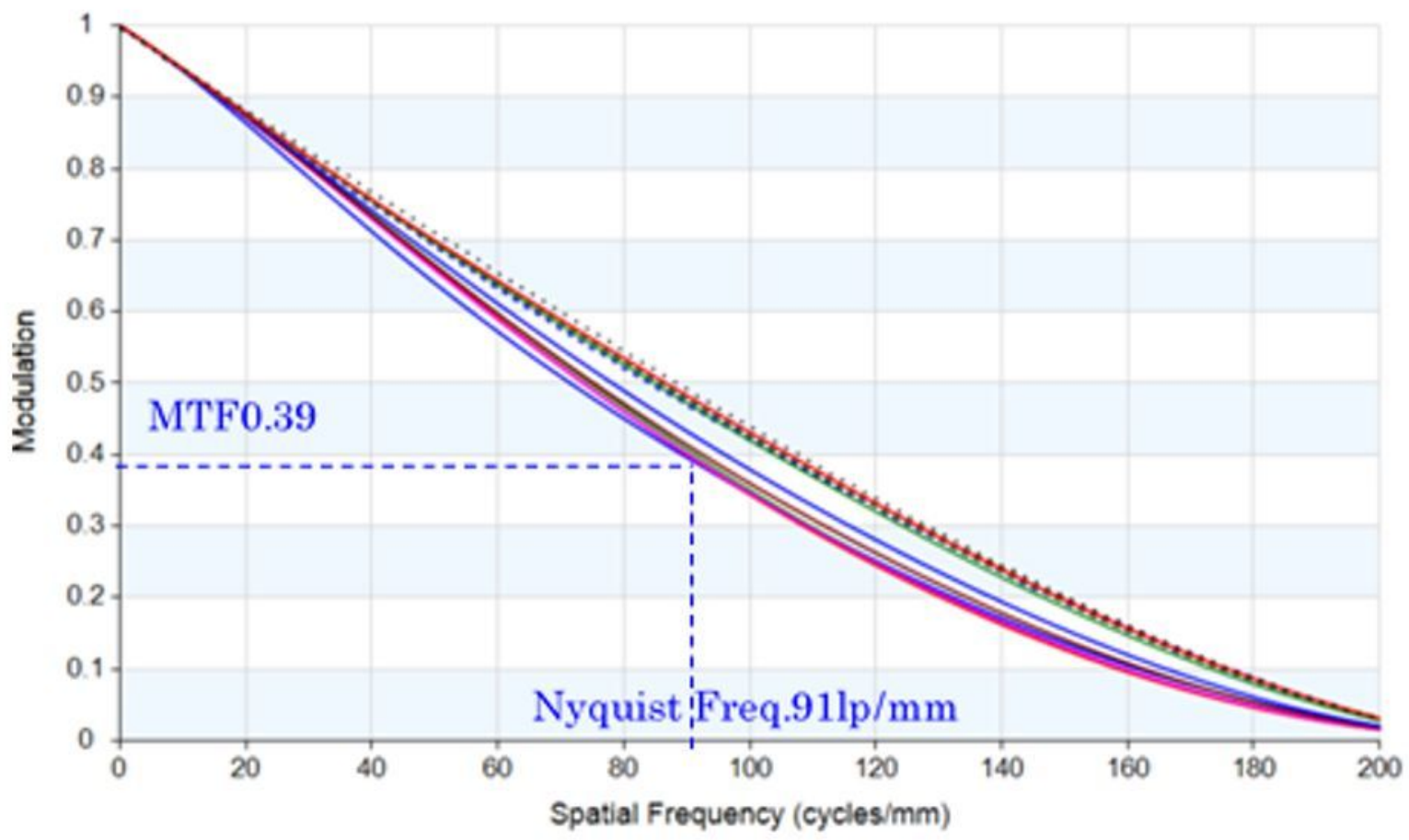

* F1: Diff. Limit

-F1: (RIH) $0.000 \mathrm{~mm}$

- F2: T (RIH) $2.404 \mathrm{~mm}$

- F2: R (RIH) $2.404 \mathrm{~mm}$

- F3: T (RIH) $4.808 \mathrm{~mm}$

- F3: R (RIH) $4.808 \mathrm{~mm}$

- F4: T (RIH) $5.769 \mathrm{~mm}$

. F4: R (RIH) $5.769 \mathrm{~mm}$

- F5: T (RIH) $6.798 \mathrm{~mm}$

. F5: R (RIH) $6.798 \mathrm{~mm}$

- F6: T (RIH) $7.692 \mathrm{~mm}$

- F6: R (RIH) $7.692 \mathrm{~mm}$

- F7: T (RIH) $8.654 \mathrm{~mm}$

.FF: R (RIH) $8.654 \mathrm{~mm}$

- F8: T (RIH) $9.615 \mathrm{~mm}$

- F8: R (RIH) $9.615 \mathrm{~mm}$

\section{Figure 17}

MTF vs Spatial Freq. Design Nominal at Objective Distance 20km (Central Wavelength 730nm, Bandwidth 40nm) 


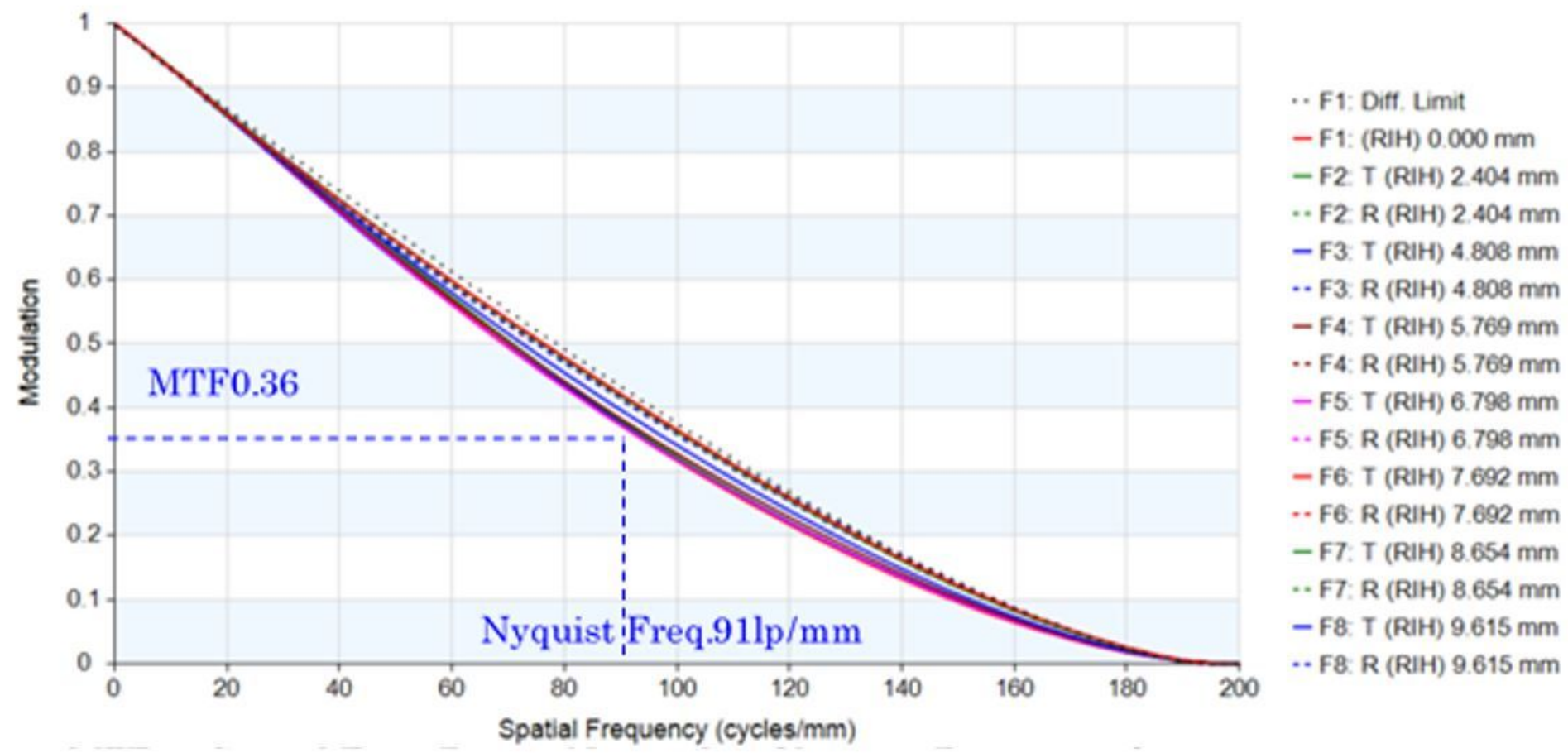

Figure 18

MTF vs Spatial Freq. Design Nominal at Objective Distance $20 \mathrm{~km}$ (Central Wavelength $860 \mathrm{~nm}$, Bandwidth 40nm)

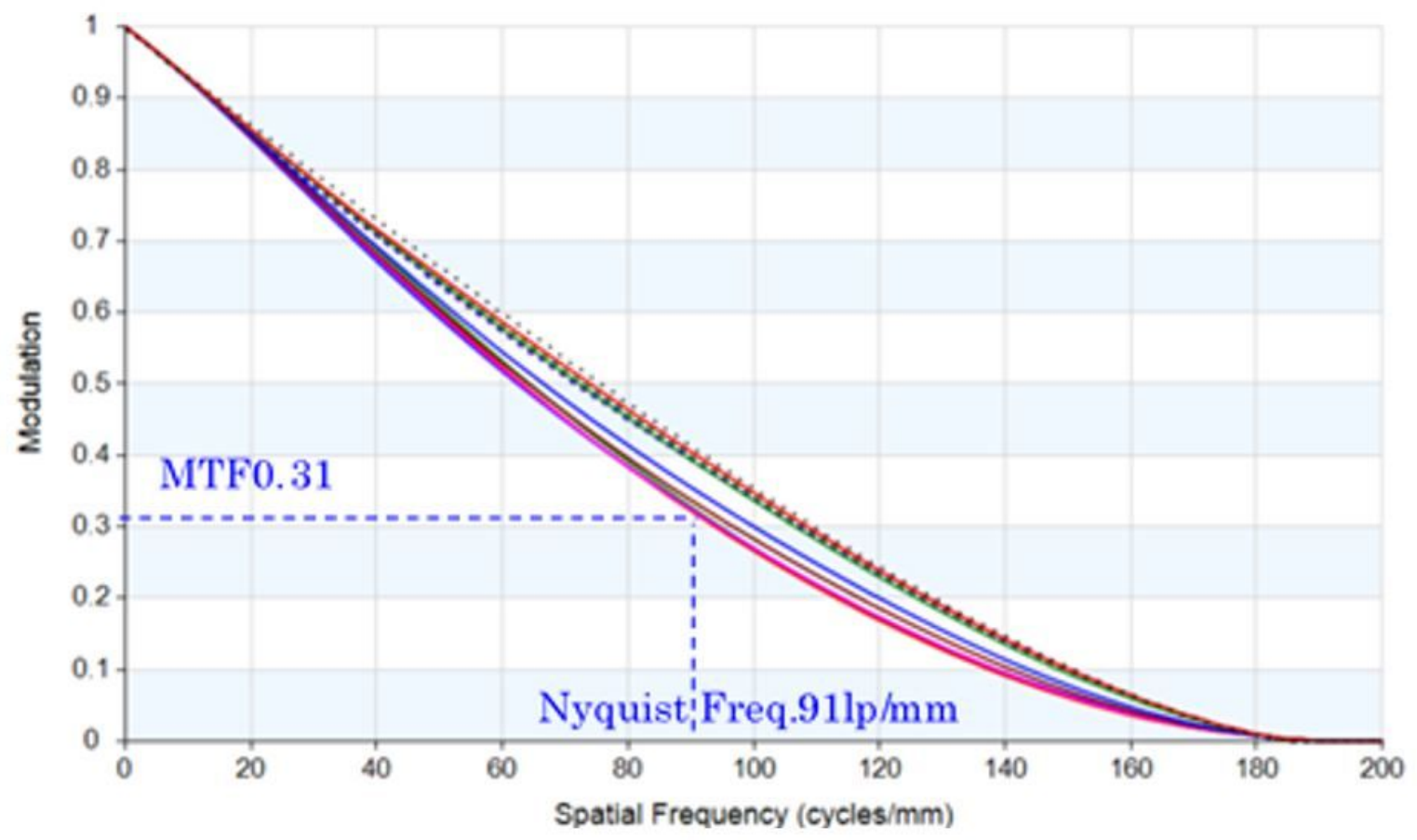

. F1: Diff. Limit

- F1: (RIH) $0.000 \mathrm{~mm}$

- F2. T (RIH) $2.404 \mathrm{~mm}$

- F2. R (RIH) 2.404 mm

- F3: T (RIH) $4.808 \mathrm{~mm}$

.- F3: R (RIH) $4.808 \mathrm{~mm}$

- F4: T (RIH) $5.769 \mathrm{~mm}$

- F4: R (RIH) $5.769 \mathrm{~mm}$

- F5: T (RIH) $6.798 \mathrm{~mm}$

*F5: R (RIH) $6.798 \mathrm{~mm}$

- F6: T (RIH) $7.692 \mathrm{~mm}$

- F6. R (RIH) $7.692 \mathrm{~mm}$

-F7: T (RIH) $8.654 \mathrm{~mm}$

- F7: R (RIH) $8.654 \mathrm{~mm}$

- F8: T (RIH) $9.615 \mathrm{~mm}$

“ F8. R (RIH) $9.615 \mathrm{~mm}$

\section{Figure 19}

MTF vs Spatial Freq. Design Nominal at Objective Distance 20km (Central Wavelength 950nm, Bandwidth 60nm) 
Figure 20

MTF vs Spatial Freq. Design Nominal at Objective Distance $0.8 \mathrm{~m}$ (Central Wavelength 550nm, Bandwidth 30nm)

\section{Supplementary Files}

This is a list of supplementary files associated with this preprint. Click to download.

- GA1.png 